\title{
MODELING MULTIPLE RISKS: HIDDEN DOMAIN OF ATTRACTION
}

\author{
ABHIMANYU MITRA AND SIDNEY I. RESNICK
}

\begin{abstract}
Hidden regular variation is a sub-model of multivariate regular variation and facilitates accurate estimation of joint tail probabilities. We generalize the model of hidden regular variation to what we call hidden domain of attraction. We exhibit examples that illustrate the need for a more general model and discuss detection and estimation techniques.
\end{abstract}

\section{INTRODUCTION}

Tail probabilities, especially joint tail probabilities, provide useful risk measures for many applications including finance [13], environmental protection [17] and hydrology [5, 3]. Multivariate extreme value theory (MEVT) is a tool to approximate such tail probabilities but in many common circumstances the tool gives an incorrect tail probability approximation of 0 . This paper points out that even when hidden regular variation (HRV) is not applicable, a more general concept called hidden domain of attraction may yield a fix.

The joint distribution $H(\cdot)$ of a bivariate random vector $\boldsymbol{X}=\left(X^{1}, X^{2}\right)$ belongs to the maximal domain of attraction of a bivariate distribution $G(\cdot)$ if there exist scaling and centering constants $a_{n}^{i}>0$ and $b_{n}^{i}, i=1,2$, such that for all continuity points $\boldsymbol{x}=\left(x^{1}, x^{2}\right)$ of $G$,

$$
\lim _{n \rightarrow \infty}\left[H\left(a_{n}^{1} x^{1}+b_{n}^{1}, a_{n}^{2} x^{2}+b_{n}^{2}\right)\right]^{n}=G\left(x^{1}, x^{2}\right)
$$

and both the marginal distributions of $G(\cdot), G^{1}(\cdot)$ and $G^{2}(\cdot)$, are non-degenerate extreme value distributions [6, page 208]. The convergence relation (1.1) is equivalent to the condition that as $n \rightarrow \infty$,

$$
n P\left[\left(\frac{X^{1}-b_{n}^{1}}{a_{n}^{1}}, \frac{X^{2}-b_{n}^{2}}{a_{n}^{2}}\right) \in \cdot\right] \stackrel{v}{\rightarrow} \nu(\cdot)
$$

in $M_{+}(\mathbb{E})$, where $\mathbb{E}=[-\infty, \infty]^{2} \backslash\{(-\infty,-\infty)\}$ or $\mathbb{E}=[0, \infty]^{2} \backslash\{(0,0)\}$ or $\mathbb{E}=[-\infty, \infty] \times[0, \infty] \backslash$ $\{(-\infty, 0)\}$ or $\mathbb{E}=[0, \infty] \times[-\infty, \infty] \backslash\{(0,-\infty)\}$ depending on the case. Also, $M_{+}(\mathbb{E})$ denotes the set of Radon measures on $\mathbb{E}$ and $\stackrel{v}{\rightarrow}$ denotes vague convergence. The limit measure $\nu(\cdot)$ in (1.2) is related to the limit distribution $G(\cdot)$ in (1.1) as follows: for $\boldsymbol{x}=\left(x^{1}, x^{2}\right) \in \mathbb{E}$,

$$
\nu\left(\left\{\left(z^{1}, z^{2}\right) \in \mathbb{E}: z^{1} \leq x^{1}, z^{2} \leq x^{2}\right\}^{c}\right)=-\log \left(G\left(x^{1}, x^{2}\right)\right) .
$$

Assuming $\left[X^{1}>u, X^{2}>v\right]$ is a rare event, that is, that $u$ and $v$ are sufficiently large, we use MEVT to approximate the joint tail probability $P\left(X^{1}>u, X^{2}>v\right)$ as

$$
P\left(X^{1}>u, X^{2}>v\right) \approx \frac{1}{n} \nu\left(\left(\frac{u-b_{n}^{1}}{a_{n}^{1}}, \infty\right] \times\left(\frac{v-b_{n}^{2}}{a_{n}^{2}}, \infty\right]\right) .
$$

However, in the presence of asymptotic independence [6, page 226], (1.4) approximates the joint tail probability $P\left(X^{1}>u, X^{2}>v\right)$ as zero. Perhaps this approximation is crude and a better estimate is possible.

Key words and phrases. Regular variation, maximal domain of attraction, spectral measure, risk sets.

S. Resnick and A. Mitra were partially supported by ARO Contract W911NF-10-1-0289 at Cornell University. 
If (1.2) holds with $\mathbb{E}=[0, \infty]^{2} \backslash\{(0,0)\}, b_{n}^{1}=b_{n}^{2}=0$ and some $a_{n}^{1}, a_{n}^{2} \uparrow \infty$, we obtain multivariate regular variation (MRV). If $X^{1}$ and $X^{2}$ are also asymptotically independent, we may improve the approximation of joint tail probabilities if hidden regular variation (HRV) is present; see Resnick [14], Mitra and Resnick [12], Resnick [15] and the seminal Ledford and Tawn [10, 11]. However, HRV requires the distribution of $X^{1} \wedge X^{2}$ to have a regularly varying tail and this may not be the case. Perhaps $X^{1} \wedge X^{2}$ has a distribution in some maximal domain of attraction other than the heavy tailed domain. In this case, HRV cannot be applied to improve joint tail probability approximation but the deficiency can be remedied by a more general approach which we call hidden domain of attraction (HDA). HRV is a special case of HDA.

If the distribution of $\boldsymbol{X}$ does not have MRV but (1.2) still holds, we may retrieve the MRV setup by transforming the components of $\boldsymbol{X}$ to $\left(U^{1}\left(X^{1}\right), U^{2}\left(X^{2}\right)\right)$, where $U^{i}(\cdot)=1 /\left(1-H^{i}(\cdot)\right)$ and $H^{i}(\cdot)$ is the distribution of $X^{i}, i=1,2$ [16, page 265]. If $X^{1}$ and $X^{2}$ are asymptotically independent, so are $\left(U^{1}\left(X^{1}\right), U^{2}\left(X^{2}\right)\right)$ and assuming $U^{1}\left(X^{1}\right) \wedge U^{2}\left(X^{2}\right)$ has a regularly varying tail, we may seek HRV. Statistically this is problematic since we do not know $U^{i}(\cdot), i=1,2$. This can be dealt with in various ways, none of which is completely satisfying or easy and a potential advantage of the notion of HDA is that it does not require that we transform components.

1.1. Outline. Section 1.2 reviews frequently used notation. In Section 2, we define hidden domain of attraction for the standard case, when both the components of the risk vector have the same distribution. Section 3 deals with HDA in the non-standard case, where we drop the identical distribution assumption for $X^{1}, X^{2}$. In both Sections 2 and 3, we exhibit examples which satisfy our model and discuss estimation procedures of limit measures that appear in the limit relations of the model. Section 4 discusses the detection techniques for HDA and estimation of joint and marginal tail probabilities. We conclude with a few remarks in Section 5 .

1.2. Notation. For simplicity, this paper is restricted to two dimensions. For denoting a vector and its components, we use:

$$
\boldsymbol{x}=\left(x^{1}, x^{2}\right), \quad x^{i}=i \text {-th component of } \boldsymbol{x}, i=1,2 .
$$

Multivariate intervals or rectangles are denoted $(\boldsymbol{x}, \boldsymbol{y}],[\boldsymbol{x}, \boldsymbol{y}]$, etc where, for instance, $(\boldsymbol{x}, \boldsymbol{y}]=$ $\left(x^{1}, y^{1}\right] \times\left(x^{2}, y^{2}\right]$. The vectors of all zeros, all ones and all infinities are denoted by $\mathbf{0}=(0,0)$, $\mathbf{1}=(1,1)$ and $\boldsymbol{\infty}=(\infty, \infty)$ respectively. We write $x^{(1)}=x^{1} \vee x^{2}, \quad x^{(2)}=x^{1} \wedge x^{2}$. So, the superscripts denote components of a vector and the ordered component is denoted by a parenthesis in the superscript.

We express vague convergence [15, page 173] of Radon measures as $\stackrel{v}{\rightarrow}$ and weak convergence of probability measures [2, page 14] as $\Rightarrow$. Denote a point measure with points $\left\{x_{i}\right\}$ in a nice space $\mathbb{F}$ by $\sum_{i} \epsilon_{x_{i}}$ where for $x \in \mathbb{F}$ and $B \subset \mathbb{F}$,

$$
\epsilon_{x}(B)= \begin{cases}1, & \text { if } x \in B \\ 0, & \text { if } x \in B^{c}\end{cases}
$$

Write $M_{+}(\mathbb{F})$ for the set of non-negative Radon measures on a space $\mathbb{F}$ topologized by the vague topology.

For a one dimensional distribution $F(x)$, set $\bar{F}:=1-F$. The inverse of a non-decreasing function $\psi(x)$ is $\psi^{\leftarrow}(x)$. 


\section{STANDARD CASE HIDDEN DOMAIN OF ATTRACTION}

Suppose that a bivariate random vector $\boldsymbol{X}=\left(X^{1}, X^{2}\right)$ with distribution $H(\boldsymbol{x})$ belongs to the maximal domain of attraction of an extreme value distribution $G$ [16, page 265], $X^{1} \stackrel{d}{=} X^{2}$ and $X^{1}$ and $X^{2}$ are asymptotically independent so that (1.1) is satisfied with $b_{n}^{1}=b_{n}^{2}=b_{n}, a_{n}^{1}=a_{n}^{2}=a_{n}>0$ and $G(x, y)=G^{1}(x) G^{2}(y)$ for $x, y \in \mathbb{R}, n \in \mathbb{N}$. Thus, (1.2) becomes

$$
n P\left[\left(\frac{\boldsymbol{X}-b_{n} \mathbf{1}}{a_{n}}\right) \in \cdot\right] \stackrel{v}{\rightarrow} \nu(\cdot)
$$

in $M_{+}(\mathbb{E})$, where $\mathbb{E}=[-\infty, \infty] \backslash\{-\infty\}$ or $\mathbb{E}=[\mathbf{0}, \infty] \backslash\{\mathbf{0}\}$. Since $G(x, y)=G^{1}(x) G^{2}(y)$ for $x, y \in \mathbb{R}$, the relation of $\nu$ and $G$ given in (1.3) gives for $\boldsymbol{x} \in \mathbb{E}$,

$$
\begin{aligned}
\nu\left(\left\{\mathbf{z} \in \mathbb{E}: z^{1} \leq x^{1}, z^{2} \leq x^{2}\right\}^{c}\right) & =-\log G^{1}\left(x^{1}\right)+-\log G^{2}\left(x^{2}\right) \\
& =\nu\left(\left\{\mathbf{z} \in \mathbb{E}: z^{1} \leq x^{1}\right\}^{c}\right)+\nu\left(\left\{\mathbf{z} \in \mathbb{E}: z^{2} \leq x^{2}\right\}^{c}\right) .
\end{aligned}
$$

The standard case contains the additional assumption that $X^{1} \stackrel{d}{=} X^{2}$, which reduces (1.2) to (2.1) and reduces possible choices for $\mathbb{E}$. The cone $\mathbb{E}=[\mathbf{0}, \boldsymbol{\infty}] \backslash\{\mathbf{0}\}$ is chosen only when $H$ has MRV.

From (2.1), the maximal component of $\boldsymbol{X}$ satisfies as $n \rightarrow \infty$,

$$
n P\left(X^{(1)}>a_{n} y+b_{n}\right) \rightarrow \nu\left(\left\{\mathbf{z} \in \mathbb{E}: z^{(1)}>y\right\}\right), \quad(y, y) \in \mathbb{E},
$$

so $X^{(1)}$ is in a maximal domain of attraction of an extreme value distribution and the distribution of $X^{(1)}$ characterizes $a_{n}$ and $b_{n}$ given in (2.1). Using one-dimensional extreme value theory, we can choose $a_{n}$ and $b_{n}$ in such a way that

$$
\psi(y):=\nu\left(\left\{\mathbf{z} \in \mathbb{E}: z^{(1)}>y\right\}\right)
$$

takes one of the following forms:

$$
\begin{aligned}
& \begin{array}{ll}
\psi(y)=\left\{\begin{array}{cl}
y^{1 / \gamma}, & \text { if } y>0, \\
0, & \text { otherwise, }
\end{array}\right. \\
\psi(y)=e^{y} & \text { if } \gamma>0,
\end{array} \\
& \psi(y)=\left\{\begin{array}{ccc}
\infty, & \text { if } y>0, & \text { if } \gamma<0, \\
(-y)^{-1 / \gamma}, & \text { otherwise, }
\end{array}\right.
\end{aligned}
$$

where $\gamma$ is the extreme value index of the distribution of $X^{(1)}$ [16, page 9]. This remains our standing assumption for the following discussion.

We define a sub-model of MEVT called (standard case) hidden domain of attraction (HDA). HDA helps approximate joint tail probabilities in the presence of asymptotic independence and includes HRV as a special case. If $\mathbb{E}$ is either $[-\infty, \infty] \backslash\{-\infty\}$ or $[\mathbf{0}, \infty] \backslash\{\mathbf{0}\}$, define $\mathbb{E}^{0}$ as $(-\infty, \infty]$ or $(\mathbf{0}, \infty]$ but see Remark 2.2(2) before jumping to erroneous conclusions that $\mathbb{E}^{0} \subset \mathbb{E}$ is always true.

Definition 2.1. The distribution of $\boldsymbol{X}=\left(X^{1}, X^{2}\right)$ has standard case hidden domain of attraction on the cone $\mathbb{E}^{0}$ if (i) $X^{1} \stackrel{d}{=} X^{2}$; (ii) (2.1) and (2.2) hold; (iii) there exist positive scaling and real centering constants $\left\{c_{n}\right\}$ and $\left\{d_{n}\right\}$ and a non-zero measure $\nu^{0} \in M_{+}\left(\mathbb{E}^{0}\right)$ such that in $M_{+}\left(\mathbb{E}^{0}\right)$,

$$
n P\left[\left(\boldsymbol{X}-d_{n} \mathbf{1}\right) / c_{n} \in \cdot\right] \stackrel{v}{\rightarrow} \nu^{0}(\cdot) \quad(n \rightarrow \infty) .
$$

We emphasize that the definition requires that $X^{1} \stackrel{d}{=} X^{2}$ and that the distribution of $\boldsymbol{X}$ belongs to the maximal domain of attraction of an extreme value product measure $G$ with exponent measure $\nu$. Some other remarks: 
Remark 2.2. (1) Hidden regular variation assumes (2.1) is satisfied on the cone $\mathbb{E}=[\mathbf{0}, \infty] \backslash\{\mathbf{0}\}$ with $b_{n}=0$ and $a_{n} \uparrow \infty$ and (2.5) is satisfied on the cone $\mathbb{E}^{0}=(\mathbf{0}, \infty]$ with $d_{n}=0$ and $c_{n} \uparrow \infty$. Moreover, $a_{n} / c_{n} \rightarrow \infty$ as $n \rightarrow \infty$. Hidden regular variation is a special case of hidden domain of attraction. HRV is the only sub-model of HDA where the cone $\mathbb{E}^{0}$ in $(2.5)$ is $\mathbb{E}^{0}=(\mathbf{0}, \infty]$.

(2) From (2.5) the minimum component of $\boldsymbol{X}$ satisfies,

$$
n P\left[X^{(2)}>c_{n} y+d_{n}\right] \rightarrow \nu^{0}((y, \infty] \times(y, \infty]) \quad\left((y, y) \in \mathbb{E}^{0}\right),
$$

and therefore, the distribution of $X^{(2)}$ belongs to the maximal domain of attraction of an extreme value distribution [6, page 4]. When HRV exists, the distribution of $X^{(2)}$ has a regularly varying tail and is hence in the domain of attraction of the Fréchet distribution. HDA allows the additional cases where the distribution of $X^{(2)}$ belongs to the domain of attraction of the Gumbel or the Weibull distribution.

The distribution of $X^{(2)}$ determines the scaling and centering constants $\left\{c_{n}\right\}$ and $\left\{d_{n}\right\}$ and the cone $\mathbb{E}^{0}$. As illustrated by Example 2.7, even if $\mathbb{E}=[\mathbf{0}, \boldsymbol{\infty}] \backslash\{\mathbf{0}\}$, the cone $\mathbb{E}^{0}$ could be $(-\infty, \infty]$ and $\mathbb{E}^{0}$ is not necessarily a sub-cone of $\mathbb{E}$, as was the case for HRV [14].

(3) From (2.3), we get that $X^{(1)}$ belongs to the maximal domain of attraction of some extreme value distribution. Since $X^{(1)} \geq X^{(2)}$, the convergence relation (2.1) puts some restriction as to what possible convergences can hold in (2.5). For example, if (2.1) is satisfied with $X^{(1)}$ being in the Gumbel domain of attraction, then HRV can never hold on $\mathbb{E}^{0}$ since the tail of $X^{(2)}$ cannot be heavier than the tail of $X^{(1)}$.

2.1. Semi-parametric structure of $\nu^{0}$. The limit measure $\nu^{0}$ in (2.5) has a semi-parametric structure which assists estimation (as in [12] for HRV) and which characterizes the class of possible limit measures as a class indexed by a real parameter and a set of probability measures.

To understand this semi-parametric structure, proceed as follows. Let $H^{(2)}(\cdot)$ be the distribution of $X^{(2)}$ and define the function $\psi^{0}(\cdot)$ as

$$
\psi^{0}(y):=\left[\nu^{0}((y, \infty] \times(y, \infty])\right]^{-1} .
$$

where $\nu^{0}(\cdot)$ is given in (2.5). From (2.6) we get

$$
n \overline{H^{(2)}}\left(c_{n} y+d_{n}\right) \rightarrow\left[\psi^{0}(y)\right]^{-1} \quad(y \in \mathbb{R}) .
$$

Hence, from the one-dimensional extreme value theory, $H^{(2)}$ is in a maximal domain of attraction. Let $\gamma^{0}$ be the extreme value index of $H^{(2)}$. Assuming $c_{n}$ and $d_{n}$ are chosen suitably [16, page 9], $\psi^{0}(\cdot)$ must take one of the following three forms:

$$
\begin{aligned}
& \psi^{0}(y)=\left\{\begin{array}{cc}
y^{1 / \gamma^{0}}, & \text { if } y>0, \\
0, & \text { otherwise, }
\end{array} \quad \text { if } \gamma^{0}>0,\right. \\
& \psi^{0}(y)=e^{y}, \quad \text { if } \gamma^{0}=0, \\
& \psi^{0}(y)=\left\{\begin{array}{cc}
\infty, & \text { if } y>0, \\
(-y)^{-1 / \gamma^{0}}, & \text { otherwise, }
\end{array} \quad \text { if } \gamma^{0}<0 .\right.
\end{aligned}
$$

Henceforth assume that $c_{n}$ and $d_{n}$ are chosen so that (2.9) is true. Define

$$
U^{(2)}(x)=1 /\left(1-H^{(2)}(x)\right),
$$

and the following helps us identify the semi-parametric structure of $\nu^{0}$. 
Proposition 2.3. The convergence in (2.5) that defines HDA is equivalent to the regular variation on $(\mathbf{0}, \infty]$,

$$
n P\left[n^{-1}\left(U^{(2)}\left(X^{1}\right), U^{(2)}\left(X^{2}\right)\right) \in \cdot\right] \stackrel{v}{\rightarrow} \tilde{\nu}^{0}(\cdot)
$$

where $U^{(2)}(\cdot)$ is defined in (2.10) and $\tilde{\nu}^{0}(\cdot)$ is a Radon measure on $(\mathbf{0}, \infty]$ that is related to the limit measure $\nu^{0}(\cdot)$ in (2.5) by

$$
\tilde{\nu}^{0}\left(\left(x^{1}, \infty\right] \times\left(x^{2}, \infty\right]\right)=\nu^{0}\left(\left(\left(\psi^{0}\right)^{\leftarrow}\left(x^{1}\right), \infty\right] \times\left(\left(\psi^{0}\right)^{\leftarrow}\left(x^{2}\right), \infty\right]\right), \quad(\boldsymbol{x} \in(\mathbf{0}, \infty]) .
$$

The measure $\tilde{\nu}^{0}(\cdot)$ satisfies the scaling property:

$$
\tilde{\nu}^{0}(c \cdot)=c^{-1} \tilde{\nu}^{0}(\cdot), \quad c>0 .
$$

Remark 2.4. (i) Proposition 2.5 below shows that the limit measure $\tilde{\nu}^{0}$ is determined by a probability measure $S^{0}$. Thus the family of limits in (2.11) is indexed by probability measures and Proposition 2.3 shows that $\nu^{0}$ has semi-parametric structure: the probability measure $S^{0}$ determines $\tilde{\nu}^{0}$ and given $\gamma^{0}$, we get $\psi^{0}(\cdot)$ from (2.9) and then applying (2.12), we get $\nu^{0}$.

(ii) If the support of the distribution of $X^{(2)}$ is smaller than that of $X^{i}, i=1,2$, then $U^{(2)}\left(X^{i}\right)$ could take the value $\infty$ with positive probability. Hence, in the following discussion, we treat $U^{(2)}\left(X^{i}\right), i=1,2$, as extended random variables.

Proof of Proposition [2.3. To see that (2.11) implies (2.5), observe that for $\boldsymbol{x} \in \mathbb{E}^{0}$,

$$
\begin{aligned}
n P\left[\frac{X^{1}-d_{n}}{c_{n}}>x^{1},\right. & \left.\frac{X^{2}-d_{n}}{c_{n}}>x^{2}\right] \\
& =n P\left[\frac{U^{(2)}\left(X^{1}\right)}{n}>\frac{U^{(2)}\left(c_{n} x^{1}+d_{n}\right)}{n}, \frac{U^{(2)}\left(X^{2}\right)}{n}>\frac{U^{(2)}\left(c_{n} x^{2}+d_{n}\right)}{n}\right] \\
& \rightarrow \tilde{\nu}^{0}\left(\left(\psi^{0}\left(x^{1}\right), \infty\right] \times\left(\psi^{0}\left(x^{2}\right), \infty\right]\right)=\nu^{0}\left(\left(x^{1}, \infty\right] \times\left(x^{2}, \infty\right]\right),
\end{aligned}
$$

where the convergence follows from (2.8) and (2.11) and the last equality follows from (2.12) and the forms of $\psi^{0}(\cdot)$ given in (2.9). Hence, (2.5) holds. The converse is similar and is omitted.

The scaling property (2.13) implies that we can express (2.11) in an alternate coordinate system that transforms the limit measure into a product. From Proposition [2.3, if the distribution of $\boldsymbol{X}$ satisfies Definition 2.1 and has HDA, then $\left(U^{(2)}\left(X^{1}\right), U^{(2)}\left(X^{2}\right)\right)$ has regular variation on $(\mathbf{0}, \infty]$. So, using (2.7), (2.9) and (2.12), we get that

$$
\tilde{\nu}^{0}((\mathbf{1}, \boldsymbol{\infty}])=\nu^{0}\left(\left(\left(\psi^{0}\right)^{\leftarrow}(1), \infty\right]^{2}\right)=\left[\psi^{0}\left(\left(\psi^{0}\right)^{\leftarrow}(1)\right)\right]^{-1}=1 .
$$

This plus the scaling property (2.13) implies $\tilde{\nu}^{0}([\mathbf{1}, \boldsymbol{\infty}])=1$. This, Proposition 3.1 of [12] and Proposition 2.3 yield the equivalent convergence in alternate coordinates given in (2.14) below.

For Proposition [2.5, we need the following: let $\nu_{1}$ be a Pareto measure on $(0, \infty]$ satisfying $\nu_{1}((y, \infty])=y^{-1}$ for $y>0$. Since $U^{(2)}(\cdot)$ is non-decreasing, $U^{(2)}\left(X^{(2)}\right)=U^{(2)}\left(X^{1}\right) \wedge U^{(2)}\left(X^{2}\right)$. Set

$$
\delta \aleph^{(2)}=\left\{\boldsymbol{x} \in(0, \infty]^{2}: x^{(2)}=1\right\} .
$$

Proposition 2.5. The convergence in 2.11) is equivalent to

$$
n P\left[\left(\frac{U^{(2)}\left(X^{(2)}\right)}{n},\left(\frac{U^{(2)}\left(X^{1}\right)}{U^{(2)}\left(X^{(2)}\right)}, \frac{U^{(2)}\left(X^{2}\right)}{U^{(2)}\left(X^{(2)}\right)}\right)\right) \in \cdot\right] \stackrel{v}{\rightarrow} \nu_{1} \times S^{0}(\cdot),
$$


on $(0, \infty] \times \delta \aleph^{(2)}$, where $S^{0}$ is a probability measure on $\delta \aleph^{(2)}$. The relation between $\tilde{\nu}^{0}$ in (2.11) and $S^{0}$ is

$$
\tilde{\nu}^{0}\left(\left\{\boldsymbol{x} \in(0, \infty]^{2}: x^{(2)} \geq r, \boldsymbol{x} / x^{(2)} \in \Lambda\right\}\right)=r^{-1} S^{0}(\Lambda),
$$

for $r>0$ and Borel sets $\Lambda \subset \delta \aleph^{(2)}$.

We call the probability measure $S^{0}$ standardized hidden spectral measure.

HRV is a special case of HDA. For HRV, a similar spectral measure $S^{(2)}(\cdot)$ is defined [12] on $\delta \aleph^{(2)}$ called the hidden spectral measure by

$$
P\left[\boldsymbol{X} / X^{(2)} \in \cdot \mid X^{(2)}>t\right] \Rightarrow S^{(2)}(\cdot) \quad(t \rightarrow \infty) .
$$

The standardized hidden spectral measure $S^{0}(\cdot)$ on $\delta \aleph^{(2)}$ given in (2.14) is

$$
P\left[\frac{\left(U^{(2)}\left(X^{1}\right), U^{(2)}\left(X^{2}\right)\right)}{U^{(2)}\left(X^{(2)}\right)} \in \cdot \mid X^{(2)}>t\right] \Rightarrow S^{0}(\cdot),
$$

where the convergence holds as $t \rightarrow x_{H^{(2)}}=\sup \left\{y \in \mathbb{R}: H^{(2)}(y)<1\right\}$. If HRV exists, $x_{H^{(2)}}=\infty$.

2.2. Examples. We give examples of distributions that possess multivariate regular variation with asymptotic independence. Each has hidden domain of attraction but not hidden regular variation emphasizing the need for a concept beyond HRV.

Example 2.6. Suppose, $W_{1}, W_{2} \stackrel{i i d}{\sim} F(\cdot)$ and $Z_{1}, Z_{2} \stackrel{i i d}{\sim} D(\cdot)$, where $F$ and $D$ belong to the maximal domains of attraction of the Fréchet with index $\alpha=1$ and Gumbel distributions respectively. Let $B$ be a Bernoulli random variable such that $P[B=1]=0.5=1-P[B=0]$. Assume the random variables $W_{1}, W_{2}, Z_{1}, Z_{2}$ and $B$ are mutually independent and define a bivariate random vector $\boldsymbol{X}$ as

$$
\boldsymbol{X}=\left(X^{1}, X^{2}\right)=B\left(W_{1}, Z_{1}\right)+(1-B)\left(Z_{2}, W_{2}\right) .
$$

We show that the distribution of $\boldsymbol{X}$ has MRV. It suffices [4] to verify that $t_{1} X^{1} \vee t_{2} X^{2}$ has a regularly varying tail for any $t_{1}, t_{2}>0$. Since $F$ has a regulary varying tail and

$$
P\left[t_{1} W_{1} \vee t_{2} Z_{1}>x\right] \sim P\left[t_{1} W_{1}>x\right], \quad(x \rightarrow \infty),
$$

$t_{1} X^{1} \vee t_{2} X^{2}$ also has a regularly varying tail. Thus for appropriate $a_{n} \uparrow \infty$,

$$
n P\left[\boldsymbol{X} / a_{n} \in \cdot\right] \stackrel{v}{\rightarrow} \nu(\cdot)
$$

on $\mathbb{E}=[0, \infty]^{2} \backslash\{\mathbf{0}\}$, where $\nu\left(\left(\left[0, x^{1}\right] \times\left[0, x^{2}\right]\right)^{c}\right)=\frac{1}{2}\left(\left(x^{1}\right)^{-1}+\left(x^{2}\right)^{-1}\right)$. Therefore, the distribution of $\boldsymbol{X}$ has MRV with asymptotic independence on $\mathbb{E}$.

Furthermore, $X^{(2)}$ belongs to the maximal domain of attraction of a Gumbel distribution and therefore, HRV does not exist. To see this, without loss of generality [1, 16], assume that $D$ is a von-Mises function [16, page 40] and for specificity assume the right endpoint of $D$ is infinite. The form of the tail is

$$
\bar{D}(x)=c e^{-\int_{1}^{x}\left[f_{D}(y)\right]^{-1} d y} \quad \text { and } \quad f_{D}^{\prime}(x) \stackrel{x \rightarrow \infty}{\rightarrow} 0
$$

where $c>0$ is some constant. Likewise, assume without loss of generality [16, page 58] that $F$ satisfies $x F^{\prime}(x) / \bar{F}(x) \rightarrow 1$, where 1 is the index of regular variation of $\bar{F}$. Then $\overline{H^{(2)}}(x):=$ $\bar{F}(x) \bar{D}(x)$ is the tail of a von-Mises function with auxiliary function

$$
f_{H^{(2)}}(t)=t f_{D}(t) /\left(t+1 \cdot f_{D}(t)\right) \stackrel{t \rightarrow \infty}{\sim} f_{D}(t)
$$


since

$$
\frac{1}{f_{H^{(2)}}(t)}=\frac{H^{(2)^{\prime}}(t)}{\overline{H^{(2)}}(t)}=\frac{F^{\prime}(t)}{\bar{F}(t)}+\frac{D^{\prime}(t)}{\bar{D}(t)} \stackrel{t \rightarrow \infty}{\sim} t^{-1}+\frac{1}{f_{D}(t)}
$$

From [16, Corollary 1.7, page 46] $H^{(2)}$ belongs to the maximal domain of attraction of the Gumbel distribution.

Next we choose scaling and centering constants $\left\{c_{n}\right\}$ and $\left\{d_{n}\right\}$ in (2.8) so that $\psi^{0}(\cdot)=e^{y}$ (one of the forms in (2.9) $)$. The usual choices are [16, page 40] $d_{n}=\left(1 \overline{H^{(2)}}\right)^{\leftarrow}(n)$ and $c_{n}=f_{H^{(2)}}\left(d_{n}\right)$.

Now, observe that for $\boldsymbol{x} \in \mathbb{E}^{0}=(-\infty, \infty]^{2}$, as $n \rightarrow \infty$,

$$
\begin{aligned}
n P\left[X^{1}>\right. & \left.d_{n}+c_{n} x^{1}, X^{2}>d_{n}+c_{n} x^{2}\right] \\
= & \frac{n}{2} \bar{F}\left(d_{n}+c_{n} x^{1}\right) \bar{D}\left(d_{n}+c_{n} x^{2}\right)+\frac{n}{2} \bar{F}\left(d_{n}+c_{n} x^{2}\right) \bar{D}\left(d_{n}+c_{n} x^{1}\right) \\
= & \frac{n}{2}\left(\frac{\bar{F}\left(d_{n}+c_{n} x^{1}\right)}{\bar{F}\left(d_{n}+c_{n} x^{2}\right)}\right) \overline{H^{(2)}}\left(d_{n}+c_{n} x^{2}\right)+\frac{n}{2}\left(\frac{\bar{F}\left(d_{n}+c_{n} x^{2}\right)}{\bar{F}\left(d_{n}+c_{n} x^{1}\right)}\right) \overline{H^{(2)}}\left(d_{n}+c_{n} x^{1}\right) \\
& \rightarrow \frac{1}{2}\left(e^{-x^{1}}+e^{-x^{2}}\right) .
\end{aligned}
$$

The convergence follows from the facts that $\bar{F}$ is regularly varying, $c_{n} / d_{n} \rightarrow 0$ and (2.8) holds with $\psi^{0}(y)=e^{y}$. Therefore, as in Definition [2.1, the distribution of $\boldsymbol{X}$ has HDA on $\mathbb{E}^{0}=(-\infty, \infty]^{2}$ with limit measure $\nu^{0}$ such that for $\boldsymbol{x}=\left(x^{1}, x^{2}\right) \in \mathbb{E}^{0}$,

$$
\nu^{0}\left(\left(x^{1}, \infty\right] \times\left(x^{2}, \infty\right]\right)=\frac{1}{2}\left(e^{-x^{1}}+e^{-x^{2}}\right) .
$$

Thus the distribution of $\boldsymbol{X}$ is regularly varying on $\mathbb{E}$, has HDA on $\mathbb{E}^{0}$, but does not have HRV since HRV requires the distribution of $X^{(2)}$ to be in the domain of attraction of the Fréchet distribution [16, page 54].

Example 2.7. Suppose, $U \sim$ Uniform $([0,1])$. Define the random vector $\boldsymbol{X}$ as

$$
\boldsymbol{X}=\left(X^{1}, X^{2}\right)=\left(\frac{1}{U}, \frac{1}{1-U}\right) .
$$

Now, note that for $x^{1}, x^{2}>0,2 n>\left(x^{1}\right)^{-1}+\left(x^{2}\right)^{-1}$,

$$
\begin{aligned}
n(1-P[1 / U & \left.\left.\leq 2 n x^{1}, 1 /(1-U) \leq 2 n x^{2}\right]\right)=n\left(1-P\left[U \geq\left(2 n x^{1}\right)^{-1}, U \leq 1-\left(2 n x^{2}\right)^{-1}\right]\right) \\
& =n\left(1-\left(1-\left(2 n x^{2}\right)^{-1}-\left(2 n x^{1}\right)^{-1}\right)\right) \rightarrow \frac{1}{2}\left(\left(x^{1}\right)^{-1}+\left(x^{2}\right)^{-1}\right),
\end{aligned}
$$

as $n \rightarrow \infty$. Therefore, on $\mathbb{E}=[0, \infty]^{2} \backslash\{\mathbf{0}\}, n P[\boldsymbol{X} / 2 n \in \cdot] \stackrel{v}{\rightarrow} \nu(\cdot)$ where the limit measure $\nu$ satisfies $\nu\left(\left(\left[0, x^{1}\right] \times\left[0, x^{2}\right]\right)^{c}\right)=\left(\left(x^{1}\right)^{-1}+\left(x^{2}\right)^{-1}\right) / 2$, for $\boldsymbol{x} \in \mathbb{E}$ and thus the distribution of $\boldsymbol{X}$ has MRV with asymptotic independence.

Also note that for $\left\{\left(x^{1}, x^{2}\right) \in(-\infty, \infty]^{2}: x^{1}+x^{2} \leq 0\right\}$, and large $n$,

$$
\begin{aligned}
n P\left[X^{1}\right. & \left.>2+\frac{2 x^{1}}{n+1}, X^{2}>2+\frac{2 x^{2}}{n+1}\right]=n P\left[U<\frac{n+1}{2 n+2+2 x^{1}}, U>\frac{n+1+2 x^{2}}{2 n+2+2 x^{2}}\right] \\
& =n\left(\frac{n+1}{2 n+2+2 x^{1}}-\frac{n+1+2 x^{2}}{2 n+2+2 x^{2}}\right)=n\left(\frac{1}{2}-\frac{x^{1}}{2 n+2+2 x^{1}}-\frac{1}{2}-\frac{x^{2}}{2 n+2+2 x^{2}}\right) \\
& \rightarrow \frac{1}{2}\left(\left(-x^{1}\right)+\left(-x^{2}\right)\right),
\end{aligned}
$$


as $n \rightarrow \infty$. Similar calculations show that for $\left\{\left(x^{1}, x^{2}\right) \in(-\infty, \infty]^{2}: x^{1}+x^{2}>0\right\}$,

$$
n P\left[X^{1}>2+\frac{2 x^{1}}{n+1}, X^{2}>2+\frac{2 x^{2}}{n+1}\right] \rightarrow 0,
$$

as $n \rightarrow \infty$. Therefore, the distribution of $\boldsymbol{X}$ has HDA as in Definition 2.1 on $\mathbb{E}^{0}=(-\infty, \infty]^{2}$ with limit measure $\nu^{0}$ such that for $\boldsymbol{x} \in \mathbb{E}^{0}$,

$$
\nu^{0}\left(\left(x^{1}, \infty\right] \times\left(x^{2}, \infty\right]\right)=\left\{\begin{array}{lr}
\frac{1}{2}\left(\left(-x^{1}\right)+\left(-x^{2}\right)\right), & \text { if } x^{1}+x^{2} \leq 0 \\
0 & \text { otherwise }
\end{array}\right.
$$

From (2.17) and (2.18) it also follows that $X^{(2)}$ belongs to the domain of attraction of the reversed Weibull distribution [16, page 59]. So, the distribution of $\boldsymbol{X}$ has HDA, but does not have HRV and furthermore, $\mathbb{E}^{0}$ is not a subset of $\mathbb{E}$.

2.3. Estimation. Because the marginal distributions are assumed to be the same, the standard case is somewhat unrealistic for applications but it is important to understand estimation for this case before moving on to more realistic scenarios.

To estimate joint tail probabilities, we first estimate the limit measure $\nu^{0}$ given in (2.5). Let, $\left\{\boldsymbol{X}, \boldsymbol{X}_{i}, i=1,2, \cdots, n\right\}$ be iid with a common distribution satisfying (2.5). From (2.5),

$$
\frac{1}{k} \sum_{i=1}^{n} \epsilon_{\left(\frac{X_{i}-d(n / k) 1}{c(n / k)}\right)}(\cdot) \Rightarrow \nu^{0}(\cdot) \quad(k \rightarrow \infty, n / k \rightarrow \infty),
$$

in $M_{+}\left(\mathbb{E}^{0}\right)$ [15, page 139]. From (2.6), the distribution of $X^{(2)}$ determines $c_{n}$ and $d_{n}$. The iid data $\left\{X_{i}^{(2)}: i=1,2, \cdots, n\right\}$ allow estimates ([6], [15, page 93]) of $c(n / k)$ and $d(n / k)$, denoted by $\hat{c}(n / k)$ and $\hat{d}(n / k)$, satisfying

$$
\frac{c(n / k)}{\hat{c}(n / k)} \stackrel{P}{\rightarrow} 1, \quad \frac{d(n / k)-\hat{d}(n / k)}{c(n / k)} \stackrel{P}{\rightarrow} 0 ;
$$

Therefore, we get the joint convergence

$$
\left(\frac{1}{k} \sum_{i=1}^{n} \epsilon\left(\frac{\boldsymbol{x}_{i}-d(n / k) \mathbf{1}}{c(n / k)}\right), \frac{d(n / k)-\hat{d}(n / k)}{c(n / k)}, \frac{c(n / k)}{\hat{c}(n / k)}\right) \Rightarrow\left(\nu^{0}(\cdot), 0,1\right)
$$

in $M_{+}\left(\mathbb{E}^{0}\right) \times \mathbb{R}^{2}$. Apply the almost surely continuous map $(\nu(\cdot), b, a) \mapsto \nu(a[(\cdot)+b \mathbf{1}])$ in (2.21) and we get the following proposition:

Proposition 2.8. Let, $\left\{\boldsymbol{X}, \boldsymbol{X}_{i}, i \geq 1\right\}$ be iid with common distribution satisfying (2.5). Then,

$$
\widehat{\nu_{n}^{0}}(\cdot):=\frac{1}{k} \sum_{i=1}^{n} \epsilon_{\left(\frac{x_{i}-\hat{d}(n / k) \mathbf{1}}{\hat{c}(n / k)}\right)}(\cdot) \Rightarrow \nu^{0}(\cdot) \quad(k \rightarrow \infty, n / k \rightarrow \infty),
$$

in $M_{+}\left(\mathbb{E}^{0}\right)$.

Estimation of $\nu^{0}(\cdot)$ in Proposition 2.8 does not exploit the semi-parametric structure discussed in Section 2.1 and has the disadvantages that (a) there is no guarantee the estimator $\widehat{\nu_{n}^{0}}(\cdot)$ is even a member of the class of possible limit measures; and (b) we are required to estimate $c(\cdot)$ and $d(\cdot)$. These disadvantages are overcome using the semi-parametric structure as was done for HRV in [12]. We need to estimate the the extreme value index $\gamma^{0}$ of the distribution of $X^{(2)}$ as well as the standardized hidden spectral measure $S^{0}$. Since $\left\{X_{i}^{(2)}: i=1,2, \cdots, n\right\}$ is iid data, estimating $\gamma^{0}$ of $X^{(2)}$ is a standard procedure [6, page 65] so we concentrate on estimating $S^{0}(\cdot)$. A modification 
of a ranks method [9, 6, 6, 15] to obtain an estimator of $\tilde{\nu}^{0}(\cdot)$ avoids the need to estimate $c(\cdot)$ and $d(\cdot)$. For $i=1,2, \cdots, n$, define

$$
R_{i}^{1,(2)}=\left|\left\{j: X_{j}^{(2)} \geq X_{i}^{1}\right\}\right| \quad \text { and } \quad R_{i}^{2,(2)}=\left|\left\{j: X_{j}^{(2)} \geq X_{i}^{2}\right\}\right|,
$$

where $|\cdot|$ denotes size of a set. Note that $0 \leq R_{i}^{j,(2)} \leq n$ for $i=1,2, \cdots, n, j=1,2$. Also notice that since $R_{i}^{1,(2)} \vee R_{i}^{2,(2)}=\left|\left\{j: X_{j}^{(2)} \geq X_{i}^{(2)}\right\}\right|, 1 \leq R_{i}^{1,(2)} \vee R_{i}^{2,(2)} \leq n$ for $i=1,2, \cdots, n$. Proposition 2.9 gives an estimator of $\tilde{\nu}^{0}$ which we can modify to get an estimator of $S^{0}(\cdot)$.

Proposition 2.9. We have in $M_{+}\left((0, \infty]^{2}\right)$,

$$
\widehat{\widetilde{\nu}_{n}^{0}}(\cdot):=\frac{1}{k} \sum_{i=1}^{n} \epsilon_{\left(k / R_{i}^{1,(2)}, k / R_{i}^{2,(2)}\right)}(\cdot) \Rightarrow \tilde{\nu}^{0}(\cdot) \quad(k \rightarrow \infty, n / k \rightarrow \infty),
$$

where (2.11) defines $\tilde{\nu}^{0}(\cdot)$ and (2.23) defines $R_{i}^{1,(2)}$ and $R_{i}^{2,(2)}$.

Proof. From (2.5) and definition of $\psi^{0}(\cdot)$ given in (2.7), we have in $D((0, \infty])$,

$$
\frac{1}{k} \sum_{i=1}^{n} \epsilon\left(\left(X_{i}^{(2)}-d(n / k)\right) / c(n / k)\right)((x, \infty]) \Rightarrow\left[\psi^{0}(x)\right]^{-1} .
$$

Hence [15, page 58], inverse functions also converge in distribution in $D_{\text {left }}((0, \infty])$, the space of left continuous functions with finite right limits,

$$
\inf \left\{x: \frac{1}{k} \sum_{i=1}^{n} \epsilon_{\left(\left(X_{i}^{(2)}-d(n / k)\right) / c(n / k)\right)}((x, \infty]) \leq 1 / s\right\} \Rightarrow \inf \left\{x:\left[\psi^{0}(x)\right]^{-1} \leq 1 / s\right\}=\left(\psi^{0}\right)^{\leftarrow}(s) .
$$

Write the order statistics of $\left\{X_{1}^{(2)}, \ldots, X_{n}^{(2)}\right\}$ as $X_{(1)}^{(2)} \geq \cdots \geq X_{(n)}^{(2)}$ and observe the left side of (2.25) is

$$
\inf \left\{x: \sum_{i=1}^{n} \epsilon_{\left(\left(X_{i}^{(2)}-d(n / k)\right) / c(n / k)\right)}((x, \infty]) \leq k / s\right\}=\left(\frac{X_{(\lceil k / s\rceil)}^{(2)}-d(n / k)}{c(n / k)}\right) .
$$

From (2.19), (2.25) and (2.26) we get that as $k \rightarrow \infty$ and $n / k \rightarrow \infty$,

$$
\begin{gathered}
\left(\frac{1}{k} \sum_{i=1}^{n} \epsilon\left(\frac{\boldsymbol{X}_{i-d(n / k) 1}}{c(n / k)}\right)(\cdot), \frac{\left.X_{(\lceil k / s\rceil)}^{(2)}\right)-d(n / k)}{c(n / k)}, \frac{\left.X_{(\lceil k / t\rceil)}^{(2)}\right)-d(n / k)}{c(n / k)}\right) \\
\Rightarrow\left(\nu^{0}(\cdot),\left(\left(\psi^{0}\right)^{\leftarrow}(s),\left(\psi^{0}\right)^{\leftarrow}(t)\right)\right)
\end{gathered}
$$

in $M_{+}\left(\mathbb{E}^{0}\right) \times D_{\text {left }}((0, \infty]) \times D_{\text {left }}((0, \infty])$. Using the scaling technique as in Resnick [15, page 311] we get from (2.27) that as $k \rightarrow \infty$ and $n / k \rightarrow \infty$,

$$
\frac{1}{k} \sum_{i=1}^{n} 1_{\left\{X_{i}^{1}>X_{(\lceil k / s])}^{(2)}, X_{i}^{2}>X_{(\lceil k / t])}^{(2)}\right\}} \Rightarrow \nu^{0}\left(\left(\left(\psi^{0}\right)^{\leftarrow}(s), \infty\right] \times\left(\left(\psi^{0}\right)^{\leftarrow}(t), \infty\right]\right)=\tilde{\nu}^{0}((s, \infty] \times(t, \infty]),
$$

in $\left.D_{\text {left }}(0, \infty]\right) \times D_{\text {left }}((0, \infty])$. Since the left side of $(\underline{2.28})$ is

$$
\frac{1}{k} \sum_{i=1}^{n} 1_{\left\{R_{i}^{1,(2)}<k / s, R_{i}^{2,(2)}<k / t\right\}}=\frac{1}{k} \sum_{i=1}^{n} 1_{\left\{s<k / R_{i}^{1,(2)}, t<k / R_{i}^{2,(2)}\right\}},
$$

we have proven (2.24). 
Proposition 2.9 yields an estimator of the limit measure $\nu_{1} \times S^{0}(\cdot)$ and then an estimator of $S^{0}(\cdot)$.

Proposition 2.10. The convergence in (2.24) is equivalent to

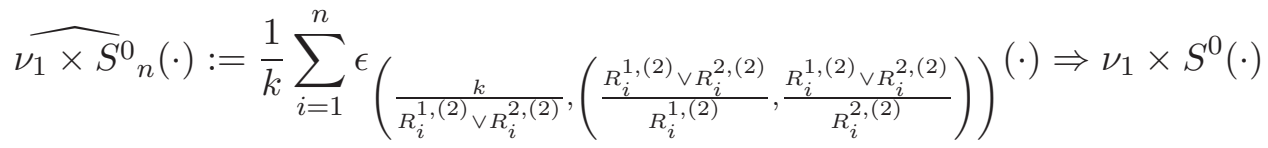

in $M_{+}\left((0, \infty] \times \delta \aleph^{(2)}\right)$, where $\nu_{1} \times S^{0}(\cdot)$ is given in Proposition 2.5.

Proof. The proof uses Proposition 2.5 and follows exactly similar steps as that of Proposition 3.7 of [12]. It is based on the map $\boldsymbol{x} \mapsto\left(x^{(2)}, \boldsymbol{x} / x^{(2)}\right)$.

From the convergence in (2.29), we construct a consistent estimator of $S^{0}(\cdot)$ :

$$
\hat{S}_{n}^{0}(\cdot):=\frac{\sum_{i=1}^{n} \epsilon\left(\frac{k}{R_{i}^{1,(2)} \vee R_{i}^{2,(2)}},\left(\frac{R_{i}^{1,(2)} \vee R_{i}^{2,(2)}}{R_{i}^{1,(2)}}, \frac{R_{i}^{1,(2)} \vee R_{i}^{2,(2)}}{R_{i}^{2,(2)}}\right)\right)^{([1, \infty] \times \cdot)}}{\sum_{i=1}^{n} \epsilon \frac{k}{R_{i}^{1,(2)} \vee R_{i}^{2,(2)}}([1, \infty])} \Rightarrow S^{0}(\cdot)
$$

in $M_{+}\left(\delta \aleph^{(2)}\right)$. Hence, we have obtained a consistent estimator for both the extreme value index $\gamma^{0}$ and the standardized hidden spectral measure $S^{0}$.

It is possible that $R_{i}^{j,(2)}=0$ for some $j=1,2$ and some $i=1,2, \cdots, n$ and thus division by zero may be indicated in (2.30). Though theoretically justified, this is not desirable when writing code for an estimator. The continuous bijection $T: \delta \aleph^{(2)} \mapsto[0,1]$ given by $T: \boldsymbol{x} \mapsto x^{2} /\left(x^{1}+x^{2}\right)$ provides an instant remedy. We use the convention that $\infty / \infty=1$ and $1 / \infty=0$. Using this transformation, (2.30) becomes

$$
\frac{\sum_{i=1}^{n} \epsilon\left(\frac{k}{R_{i}^{1,(2)} \vee R_{i}^{2,(2)}},\left(\frac{R_{i}^{1,(2)}}{R_{i}^{1,(2)}+R_{i}^{2,(2)}}\right)\right)^{([1, \infty] \times \cdot)}}{\sum_{i=1}^{n} \epsilon \frac{k}{R_{i}^{1,(2)} \vee R_{i}^{2,(2)}}([1, \infty])} \Rightarrow S^{0} \circ T^{-1}(\cdot)
$$

in $M_{+}([0,1])$. Since $T$ is a continuous bijection, we retrieve $S^{0}$ from $S^{0} \circ T^{-1}$.

\section{NON-STANDARD HidDEN DOMAIN OF ATTRACTION}

To provide more scope for applications, the non-standard case no longer assumes that $X^{1} \stackrel{d}{=} X^{2}$. However, we have found that to construct a coherent estimation theory requires careful consideration of the definitions. As in the standard case, the goal is to approximate marginal and joint tail probabilities.

3.1. How to proceed? In order for (1.2) to hold when $\boldsymbol{X}$ has different marginal distributions, one typically needs different centering and scaling constants for the two components of $\boldsymbol{X}$. Traditional theory [16, page 277, Proposition 5.15] proceeds by standardizing each component. However, a theory of hidden domain of attraction that follows this approach encounters problems in the estimation procedure that we could not resolve without strong second order conditions.

We deviate from the traditional MEVT treatment by requiring that both components in (2.1) have the same centering and scaling but permitting the limit measure to have one zero marginal. By a zero marginal, we mean that either the limit measure $\nu(\cdot)$ in (2.1) has the property

$$
\nu\left(\left\{\boldsymbol{z} \in \mathbb{E}: z^{2}>y\right\}\right)=0 \quad((y, y) \in \mathbb{E})
$$


or the same holds with $z^{1}$ in place of $z^{2}$. This could happen, for instance, if the tail of $X^{2}$ is lighter than that of $X^{1}$ or vice versa.

In the non-standard case, if we assume (2.1), the limit measure $\nu$ may satisfy:

(i) $\nu$ has a zero second marginal: for $(y, y) \in \mathbb{E}, \nu\left(\left\{z \in \mathbb{E}: z^{2}>y\right\}\right)=0$;

(ii) $\nu$ has a zero first marginal: for $(y, y) \in \mathbb{E}, \nu\left(\left\{\boldsymbol{z} \in \mathbb{E}: z^{1}>y\right\}\right)=0$,

(iii) the cases (i) and (ii) do not hold, but $\nu\left(\left\{\boldsymbol{z} \in \mathbb{E}: z^{1}>x, z^{2}>y\right\}\right)=0$ for $(x, y) \in \mathbb{E}$,

(iv) for $(x, y) \in \mathbb{E}, \nu\left(\left\{\boldsymbol{z} \in \mathbb{E}: z^{1}>x, z^{2}>y\right\}\right)>0$.

Case (iv) means (2.1) yields non-zero estimates of the marginal and joint tail probabilities, so in this case we have no need to define HDA. The definition and analysis of HDA in case (iii) is the same as the standard case discussed in Section 2. The definition and analysis of HDA are very similar for cases (i) and (ii) so focus only on case (i).

For our definition of HDA, a relevant state space is $\mathbb{E}^{\sqcap}$ where either $\mathbb{E}^{\sqcap}=[-\infty, \infty] \times(-\infty, \infty]$ or $\mathbb{E}^{\square}=[0, \infty] \times(0, \infty]$. We will see later that for case (i) it is possible to find HDA on both the cones $\mathbb{E}^{\square}$ and $\mathbb{E}^{0}$ in sequence. Thus, compared to the standard case, our estimation procedure here might involve analyzing HDA on the additional cone $\mathbb{E}^{\Gamma}$.

Definition 3.1. The distribution of $\boldsymbol{X}=\left(X^{1}, X^{2}\right)$ has hidden domain of attraction on the cone $\mathbb{E}^{\Gamma}$ if (2.1) holds with the limit measure $\nu$, the second marginal of $\nu$ is a zero measure and in addition, there exist constants $e_{n}>0$ and $f_{n} \in \mathbb{R}$ and a non-zero measure $\nu^{\sqcap} \in M_{+}\left(\mathbb{E}^{\square}\right)$ such that as $n \rightarrow \infty$,

$$
n P\left[\left(\frac{\mathbf{X}-f_{n} \mathbf{1}}{e_{n}}\right) \in \cdot\right] \stackrel{v}{\rightarrow} \nu \sqcap(\cdot) \quad \text { in } M_{+}\left(\mathbb{E}^{\sqcap}\right) .
$$

From (3.1) it follows that for $(y, y) \in \mathbb{E}^{\square}$, as $n \rightarrow \infty$,

$$
n P\left[X^{2}>e_{n} y+f_{n}\right] \rightarrow \nu^{\sqcap}\left(\left\{(u, v) \in E^{\sqcap}: v>y\right\}\right) .
$$

Therefore, the distribution of $X^{2}$, the second component of $\boldsymbol{X}$, belongs to the maximal domain of attraction of an extreme value distribution [6, page 4]. Using one-dimensional extreme value theory, $\psi \sqcap(y):=[\nu \sqcap([0, \infty] \times(y, \infty])]^{-1}$ must take one of the following forms [16, page 9$]$ :

$$
\begin{aligned}
& \psi^{\sqcap}(y)=\left\{\begin{array}{cc}
y^{1 / \gamma^{\sqcap}}, & \text { if } y>0, \\
0, & \text { otherwise, }
\end{array} \quad \text { if } \gamma^{\sqcap}>0,\right. \\
& \psi^{\sqcap}(y)=e^{y}, y \in \mathbb{R}, \quad \text { if } \gamma^{\sqcap}=0, \\
& \psi \sqcap(y)=\left\{\begin{array}{cc}
\infty, & \text { if } y>0, \\
(-y)^{-1 / \gamma^{\sqcap},} & \text { otherwise, }
\end{array} \quad \text { if } \gamma^{\sqcap}<0 .\right.
\end{aligned}
$$

The parameter $\gamma^{\sqcap}$ in (3.3) is the extreme value index of $X^{2}$. We can and always do choose $\left\{e_{n}\right\}$ and $\left\{f_{n}\right\}$ in such a way that $\psi \sqcap$ takes one of the above forms.

Remark 3.2. We make a few remarks about Definition 3.1 .

(1) Since (2.1) holds, so does (2.3) and therefore the maximum component $X^{(1)}$ belongs to the maximal domain of attraction of some extreme value distribution. Since $X^{(1)} \geq X^{2}$, the convergence relation (2.1) constrains the possible convergences in (3.1). For example, if (2.3) has $X^{(1)}$ in the Gumbel domain of attraction, then the distribution of $X^{2}$ cannot have a regularly varying tail.

(2) The distribution of $X^{2}$ determines the cone $\mathbb{E}^{\square}$ and (3.2) yields the scaling and centering constants $\left\{e_{n}\right\}$ and $\left\{f_{n}\right\}$. If the distribution of $X^{2}$ is in the Fréchet domain of attraction, $\mathbb{E}^{\sqcap}=[0, \infty] \times(0, \infty]$ and otherwise, $\mathbb{E}^{\sqcap}=[-\infty, \infty] \times(-\infty, \infty]$ 
There are two possibilities for the limit measure $\nu^{\sqcap}$ in (3.1):

(i) the limit measure $\nu^{\sqcap}$ puts zero mass on all sets $(x, \infty] \times(y, \infty]$ for $(x, y) \in \mathbb{E}^{\sqcap}$; or

(ii) the limit measure $\nu^{\sqcap}$ puts non-zero mass on one of the sets $(x, \infty] \times(y, \infty]$ for $(x, y) \in \mathbb{E}^{\sqcap}$.

The semi-parametric structure of $\nu^{\sqcap}$ discussed in the next section implies that for case (ii), $\nu^{\sqcap}((x, \infty] \times(y, \infty])>0$ for all $(x, y) \in \mathbb{E}^{\sqcap}$. So, in case (ii), we get non-zero estimates of joint tail probabilities and since we accomplished our goal there is no reason to seek further instances of HDA. However, in case (i), the measure $\nu^{\sqcap}(\cdot)$ will not provide non-zero estimates of joint tail probabilities. A potential solution is that HDA could still exist a smaller cone such as $\mathbb{E}^{0}$. The following definition formalizes the concept. For this definition, the state space is $\mathbb{E}^{0}$, where $\mathbb{E}^{0}=$ $(-\infty, \infty]^{2}$ or $\mathbb{E}^{0}=(0, \infty]^{2}$.

Definition 3.3. The distribution of $\boldsymbol{X}=\left(X^{1}, X^{2}\right)$ has hidden domain of attraction on the cones $\mathbb{E}^{\sqcap}$ and $\mathbb{E}^{0}$ if Definition 3.1 holds, the the limit measure $\nu^{\square}$ in (3.1) puts zero mass to all sets of the form $(x, \infty] \times(y, \infty]$ for $(x, y) \in \mathbb{E}^{\sqcap}$, and in addition, there exist centering and scaling constants $\left\{c_{n}\right\}$ and $\left\{d_{n}\right\}$ and a non-zero measure $\nu^{0} \in M_{+}\left(\mathbb{E}^{0}\right)$ such that as $n \rightarrow \infty$,

$$
n P\left[\left(\frac{\boldsymbol{X}-d_{n} \mathbf{1}}{c_{n}}\right) \in \cdot \stackrel{v}{\rightarrow} \nu^{0}(\cdot) \quad \text { in } M_{+}\left(\mathbb{E}^{0}\right) .\right.
$$

As noted before in (2.6), the scaling and centering constants $\left\{c_{n}\right\}$ and $\left\{d_{n}\right\}$ in (3.4) are characterized by the distribution of $X^{(2)}$, the minimum component of $\boldsymbol{X}$. Recall the definition of $\psi^{0}$ given in (2.7). As was done in the standard case discussion, we choose the scaling and centering constants $\left\{c_{n}\right\}$ and $\left\{d_{n}\right\}$ in (3.4) so that $\psi^{0}$ takes one of the forms given in (2.9). Also, whether $\mathbb{E}^{0}$ in (3.4) is $(-\infty, \infty]^{2}$ or $(0, \infty]^{2}$ is determined by the distribution of $X^{(2)}$.

3.2. Semi-parametric structure of $\nu^{\sqcap}$. Both limit measures $\nu^{\sqcap}$ of (3.1) and $\nu^{0}$ of (3.4) have semi-parametric structures. Since the semi-parametric structure of $\nu^{0}$ was discussed in Section 2.1. we concentrate only on the semi-parametric structure of $\nu^{\sqcap}$ and proceed as follows.

Recall that the distributions of $\boldsymbol{X}$ and $X^{2}$ are $H$ and $H^{2}$. Define

$$
U^{2}(x)=1 /\left(1-H^{2}(x)\right) .
$$

The following proposition relates (3.1) to a regular variation condition on $[0, \infty] \times(0, \infty]$. Its proof is similar to that of Proposition 2.3 and is omitted.

Proposition 3.4. Convergence in (3.1) is equivalent to regular variation on the cone $[0, \infty] \times(0, \infty]$,

$$
n P\left[\left(\frac{U^{2}\left(X^{1}\right)}{n}, \frac{U^{2}\left(X^{2}\right)}{n}\right) \in \cdot\right] \stackrel{v}{\rightarrow} \tilde{\nu}^{\sqcap}(\cdot) \quad\left(\text { in } M_{+}([0, \infty] \times(0, \infty]),\right.
$$

where (3.5) defines $U^{2}(\cdot)$ and $\tilde{\nu}^{\sqcap}(\cdot)$ is a Radon measure on $[0, \infty] \times(0, \infty]$. The limit measure $\tilde{\nu}^{\sqcap}(\cdot)$ is related to the limit measure in $\nu^{\sqcap}(\cdot)$ in (3.1) by the following relation: for $\left(x^{1}, x^{2}\right) \in[0, \infty] \times(0, \infty]$,

$$
\begin{aligned}
& \tilde{\nu}^{\sqcap}\left(\left(x^{1}, \infty\right] \times\left(x^{2}, \infty\right]\right)=\nu^{\sqcap}\left(\left(\left(\psi^{\sqcap}\right)^{\leftarrow}\left(x^{1}\right), \infty\right] \times\left(\left(\psi^{\sqcap}\right)^{\leftarrow}\left(x^{2}\right), \infty\right]\right), \\
& \tilde{\nu}^{\sqcap}\left(\left[0, x^{1}\right] \times\left(x^{2}, \infty\right]\right)=\nu^{\sqcap}\left(\left\{\boldsymbol{z} \in \mathbb{E}^{\sqcap}: z^{1} \leq\left(\psi^{\sqcap}\right)^{\leftarrow}\left(x^{1}\right), z^{2}>\left(\psi^{\sqcap}\right)^{\leftarrow}\left(x^{2}\right)\right\}\right) .
\end{aligned}
$$

The measure $\tilde{\nu}^{\sqcap}(\cdot)$ satisfies the scaling property:

$$
\tilde{\nu}^{\sqcap}(c \cdot)=c^{-1} \tilde{\nu}^{\sqcap}(\cdot) \quad c>0 .
$$


Remark 3.5. (i) On the semi-parametric structure of $\nu \sqcap$ : We will see that a probability measure $S^{\sqcap}$ on $[0, \infty]$ determines the limit measure $\tilde{\nu}^{\sqcap}$. The parameter $\gamma^{\sqcap}$ and the probability measure $S^{\sqcap}$ on $[0, \infty]$ determine $\nu^{\sqcap}$, since given $\gamma^{\sqcap}$ and measure $S^{\sqcap}$, we get the function $\psi^{\sqcap}(\cdot)$ in (3.3) and $\tilde{\nu}^{\sqcap}$ which through (3.7) determines $\nu \sqcap$.

(ii) If the support of the distribution of $X^{2}$ is smaller than that of $X^{1}$, then $U^{2}\left(X^{1}\right)$ could take the value $\infty$, so in this case we consider $U^{2}\left(X^{1}\right)$ as an extended random variable.

The method that shows $\tilde{\nu}^{0}\left([1, \infty]^{2}\right)=1$ also shows $\tilde{\nu}^{\sqcap}([0, \infty] \times[1, \infty])=1$. Proposition 4 of [8] and Proposition 3.4 give a convergence relation in new coordinates.

Proposition 3.6. The convergence in (3.1) is equivalent to

$$
n P\left[\left(\frac{U^{2}\left(X^{2}\right)}{n}, \frac{U^{2}\left(X^{1}\right)}{U^{2}\left(X^{2}\right)}\right) \in \cdot\right] \stackrel{v}{\rightarrow} \nu_{1} \times S^{\sqcap}(\cdot) \quad\left(\text { in } M_{+}((0, \infty] \times[0, \infty])\right),
$$

where $\nu_{1}$ is a Pareto measure on $(0, \infty]$ satisfying $\nu_{1}((x, \infty])=x^{-1}$ for $x>0$, and $S \sqcap$ is a probability measure on $[0, \infty]$, called the standardized hidden spectral measure. The relation between $\tilde{\nu} \sqcap$ given in (2.11) and $S^{\sqcap}$ is

$$
\tilde{\nu}^{\sqcap}\left(\left\{\boldsymbol{x} \in[0, \infty] \times(0, \infty]: x^{2} \geq r, x^{1} / x^{2} \in \Lambda\right\}\right)=r^{-1} S^{\sqcap}(\Lambda),
$$

which holds for all $r>0$ and all Borel sets $\Lambda \subset[0, \infty]$.

3.3. Examples. We give examples of distributions of $\boldsymbol{X}=\left(X^{1}, X^{2}\right), X^{1} \stackrel{d}{\neq} X^{2}$, and which have HDA. In Example 3.7, the limit measure $\nu^{\sqcap}$ of (3.1) puts zero mass on all sets of the form $\left(x^{1}, \infty\right] \times$ $\left(x^{2}, \infty\right]$ for $\boldsymbol{x} \in \mathbb{E}^{\square}$ and HDA also holds on $\mathbb{E}^{0}$. In Example 3.8, $\nu^{\sqcap}$ of (3.1) puts non-zero mass on sets of the form $\left(x^{1}, \infty\right] \times\left(x^{2}, \infty\right]$ for $\boldsymbol{x} \in \mathbb{E}^{\sqcap}$.

Example 3.7. Let $X^{1} \sim \exp (1), X^{2} \sim \exp (2)$ and $X^{1}$ and $X^{2}$ be independent. Then we get

$$
\begin{gathered}
n\left(1-P\left[X^{1}-\log n \leq x^{1}, X^{2}-\log n \leq x^{2}\right]\right)=n\left[1-\left(1-e^{-\left(\log n+x^{1}\right)}\right)\left(1-e^{-2\left(\log n+x^{2}\right)}\right)\right] \\
\rightarrow e^{-x^{1}} \quad \text { as } n \rightarrow \infty,
\end{gathered}
$$

which implies (2.1) holds on $\mathbb{E}=[-\infty, \infty]^{2} \backslash\{(-\infty, \infty)\}$ with $\nu\left(\left(\left[-\infty, x^{1}\right] \times\left[-\infty, x^{2}\right]\right)^{c}\right)=e^{-x^{1}}$ and $\nu$ puts mass only on $(-\infty, \infty] \times\{-\infty\}$ and $\nu$ has zero second marginal. So we seek HDA on $\mathbb{E}^{\Gamma}$. As $n \rightarrow \infty$,

$$
n P\left[X^{1}-\frac{\log n}{2} \leq x^{1}, X^{2}-\frac{\log n}{2}>x^{2}\right]=n\left(1-e^{-\left(\frac{\log n}{2}+x^{1}\right)}\right) e^{-2\left(\frac{\log n}{2}+x^{2}\right)} \rightarrow e^{-2 x^{2}},
$$

and also as $n \rightarrow \infty$,

$$
n P\left[X^{1}-\frac{\log n}{2}>x^{1}, X^{2}-\frac{\log n}{2}>x^{2}\right]=n e^{-\left(\frac{\log n}{2}+x^{1}\right)} e^{-2\left(\frac{\log n}{2}+x^{2}\right)} \rightarrow 0 .
$$

Thus, HDA exists on $\mathbb{E}^{\sqcap}=[-\infty, \infty] \times(-\infty, \infty]$ with limit measure $\nu^{\sqcap}$, where $\nu^{\sqcap}\left(\left[-\infty, x^{1}\right] \times\right.$ $\left.\left(x^{2}, \infty\right]\right)=e^{-2 x^{2}}$ and $\nu^{\sqcap}\left(\left(x^{1}, \infty\right] \times\left(x^{2}, \infty\right]\right)=0$ for $\boldsymbol{x} \in \mathbb{E}^{\square}$ so $\nu^{\sqcap}$ concentrates on $\{-\infty\} \times(-\infty, \infty]$. After peeling away both lines through $-\infty$, we look for HDA on $\mathbb{E}^{0}$. A hint for how to proceed is provided by $X^{1} \wedge X^{2} \sim \exp (3)$. Note that as $n \rightarrow \infty$,

$$
n P\left[X^{1}-\frac{\log n}{3}>x^{1}, X^{2}-\frac{\log n}{3}>x^{2}\right]=n e^{-\left(\frac{\log n}{3}+x^{1}\right)} e^{-2\left(\frac{\log n}{3}+x^{2}\right)} \rightarrow e^{-\left(x^{1}+2 x^{2}\right)} .
$$


Thus HDA exists on $\mathbb{E}^{0}=(-\infty, \infty]^{2}$ with limit measure $\nu^{0}$, where $\nu^{0}\left(\left(x^{1}, \infty\right] \times\left(x^{2}, \infty\right]\right)=e^{-\left(x^{1}+2 x^{2}\right)}$ for $\boldsymbol{x} \in \mathbb{E}^{0}$.

For this example, Definition 3.3 holds and HDA holds on both the cones $\mathbb{E}^{\square}$ and $\mathbb{E}^{0}$,

Example 3.8. Suppose $E_{1}, E_{2}, E_{3}$ are iid $\exp (1)$ random variables independent of $B \sim \operatorname{Bernoulli}(1 / 2)$ and define $\boldsymbol{X}$ as

$$
\boldsymbol{X}=B\left(E_{1}, E_{3} / 3\right)+(1-B)\left(E_{2} / 2, E_{2} / 2\right) .
$$

As $n \rightarrow \infty$,

$$
\begin{aligned}
& n\left(1-P\left[X^{1}-\log n \leq x^{1}, X^{2}-\log n \leq x^{2}\right]\right) \\
& \quad=n\left[1-\left[\frac{1}{2}\left(1-e^{-\left(\log n+x^{1}\right)}\right)\left(1-e^{-3\left(\log n+x^{2}\right)}\right)+\frac{1}{2}\left(1-e^{-2\left(\log n+x^{1} \wedge x^{2}\right)}\right)\right]\right] \rightarrow e^{-x^{1}},
\end{aligned}
$$

which implies (2.1) holds on $\mathbb{E}=[-\infty, \infty]^{2} \backslash\{(-\infty, \infty)\}$ with $\nu\left(\left(\left[-\infty, x^{1}\right] \times\left[-\infty, x^{2}\right]\right)^{c}\right)=e^{-x^{1}}$ and $\nu$ concentrates on $(-\infty, \infty] \times\{-\infty\}$. Thus $\nu$ has zero second marginal and we seek HDA on $\mathbb{E}^{\Gamma}$. Note that

$$
\begin{aligned}
& n P\left[X^{1}-\frac{\log n}{2} \leq x^{1}, X^{2}-\frac{\log n}{2}>x^{2}\right] \\
& \quad=n\left[\frac{1}{2}\left(1-e^{-\left(\frac{\log n}{2}+x^{1}\right)}\right) e^{-3\left(\frac{\log n}{2}+x^{2}\right)}+\frac{1}{2}\left(e^{-2\left(\frac{\log n}{2}+x^{2}\right)}-e^{-2\left(\frac{\log n}{2}+x^{1}\right)}\right) 1_{\left\{x^{2}<x^{1}\right\}}\right] \\
& \quad \rightarrow \frac{1}{2}\left(e^{-2 x^{2}}-e^{-2 x^{1}}\right) 1_{\left\{x^{2}<x^{1}\right\}} \quad \text { as } n \rightarrow \infty,
\end{aligned}
$$

and also

$$
\begin{aligned}
& n P\left[X^{1}-\frac{\log n}{2}>x^{1}, X^{2}-\frac{\log n}{2}>x^{2}\right] \\
& \quad=n\left[\frac{1}{2} e^{-\left(\frac{\log n}{2}+x^{1}\right)} e^{-3\left(\frac{\log n}{2}+x^{2}\right)}+\frac{1}{2} e^{-2\left(\frac{\log n}{2}+x^{1} \vee x^{2}\right)}\right] \rightarrow \frac{1}{2} e^{-2\left(x^{1} \vee x^{2}\right)} \quad \text { as } n \rightarrow \infty .
\end{aligned}
$$

Thus, HDA exists on $\mathbb{E}^{\square}=[-\infty, \infty] \times(-\infty, \infty]$ with limit measure $\nu \sqcap$, where $\nu^{\sqcap}\left(\left[-\infty, x^{1}\right] \times\right.$ $\left.\left(x^{2}, \infty\right]\right)=\frac{1}{2}\left(e^{-2 x^{2}}-e^{-2 x^{1}}\right) 1_{\left\{x^{2}<x^{1}\right\}}$ and $\nu^{\sqcap}\left(\left(x^{1}, \infty\right] \times\left(x^{2}, \infty\right]\right)=\frac{1}{2} e^{-2\left(x^{1} \vee x^{2}\right)}$ for $\boldsymbol{x} \in \mathbb{E} \sqcap$. In fact, $\nu \sqcap$ concentrates on the line $\{(x, x): x \in(-\infty, \infty)\}$.

Since $\nu^{\sqcap}\left(\left(x^{1}, \infty\right] \times\left(x^{2}, \infty\right]\right)>0$ for $\boldsymbol{x} \in \mathbb{E}^{\square}$, we do not seek HDA on $\mathbb{E}^{0}$.

3.4. Estimation methods. To estimate joint tail probabilities, we require an estimate of the limit measure $\nu^{\sqcap}$ given in Definition 3.1 and possibly $\nu^{0}$ given in Definition 3.3. Estimation of $\nu^{0}$ follows the same steps as in Section 2.3 so we concentrate on estimating $\nu \sqcap$. Let, $\left\{\boldsymbol{X}, \boldsymbol{X}_{i}, i=1,2, \cdots, n\right\}$ be iid where the distribution of $\boldsymbol{X}$ satisfies (3.1). From (3.1) we get [15), page 139] in $M_{+}\left(\mathbb{E}^{\sqcap}\right)$

$$
\frac{1}{k} \sum_{i=1}^{n} \epsilon\left(\frac{X_{i}^{1}-f(n / k)}{e(n / k)}, \frac{X_{i}^{2}-f(n / k)}{e(n / k)}\right)(\cdot) \Rightarrow \nu^{\sqcap}(\cdot) \quad(k \rightarrow \infty, n / k \rightarrow \infty) .
$$

We know from (3.2) that the distribution of $X^{2}$ characterizes $\left\{e_{n}\right\}$ and $\left\{f_{n}\right\}$ and from the iid data $\left\{X_{i}^{2}: i=1,2, \cdots, n\right\}$, we can construct estimators of $e(n / k)$ and $f(n / k)$ denoted by $\hat{e}(n / k)$ and $\hat{f}(n / k)[15$, page 93] such that

$$
\frac{e(n / k)}{\hat{e}(n / k)} \stackrel{P}{\rightarrow} 1, \quad \frac{f(n / k)-\hat{f}(n / k)}{e(n / k)} \stackrel{P}{\rightarrow} 0 .
$$


Since the limits in $(\underline{3.12})$ are constants, we get joint convergence in $M_{+}\left(\mathbb{E}^{\square}\right) \times \mathbb{R}^{2}$,

$$
\left(\frac{1}{k} \sum_{i=1}^{n} \epsilon\left(\frac{x_{i}^{1}-f(n / k)}{e(n / k)}, \frac{X_{i}^{2}-f(n / k)}{e(n / k)}\right), \frac{f(n / k)-\hat{f}(n / k)}{e(n / k)}, \frac{e(n / k)}{\hat{e}(n / k)}\right) \Rightarrow(\nu \sqcap(\cdot), 0,1)
$$

Apply the continuous mapping theorem to (3.13) using the map $(\nu(\cdot), b, a) \mapsto \nu(a[(\cdot)+b])$ to get in $M_{+}\left(\mathbb{E}^{\square}\right)$

$$
\widehat{\nu_{n}^{\Pi}}(\cdot):=\frac{1}{k} \sum_{i=1}^{n} \epsilon\left(\frac{X_{i}^{1}-\hat{f}(n / k)}{\hat{e}(n / k)}, \frac{X_{i}^{2}-\hat{f}(n / k)}{\hat{e}(n / k)}\right)(\cdot) \Rightarrow \nu^{\sqcap}(\cdot) \quad(k \rightarrow \infty, n / k \rightarrow \infty) .
$$

This estimator of $\nu \sqcap$ is non-parametric and as in Section 2.3. we exploit the semi-parametric structure of $\nu^{\sqcap}$ by estimating $\gamma^{\square}$ and the standardized hidden spectral measure $S \sqcap$. The parameter $\gamma^{\sqcap}$ is the extreme value index of the distribution of $X^{2}$ so estimating this from iid data $\left\{X_{i}^{2}\right.$ : $i=1,2, \cdots, n\}$ is standard [6, page 65]. We obtain an estimator of $S^{\sqcap}(\cdot)$ by modifying (2.23) to account for the difference between $\mathbb{E}^{0}$ and $\mathbb{E}^{\sqcap}$. Since the first step is to construct a consistent estimator of $\tilde{\nu}^{\sqcap}(\cdot)$ defined in (3.6) , define

$$
R_{i}^{1,2}:=\left|\left\{j: X_{j}^{2} \geq X_{i}^{1}\right\}\right| \quad \text { and } \quad R_{i}^{2,2}:=\left|\left\{j: X_{j}^{2} \geq X_{i}^{2}\right\}\right|, \quad(i=1,2, \cdots, n,)
$$

where $|\cdot|$ denotes size of a set. Observe $R_{i}^{2,2}$ is just the anti-rank of $X_{i}^{2}$ and thus $1 \leq R_{i}^{2,2} \leq n$ for $i=1,2, \cdots, n$. Also, $0 \leq R_{i}^{1,2} \leq n$ for $i=1,2, \cdots, n$. An estimator of $\tilde{\nu}^{\sqcap}$ is obtained from the convergence in $M_{+}([0, \infty] \times(0, \infty])$ :

$$
\widehat{\widetilde{\nu}_{n}^{\Pi}}(\cdot):=\frac{1}{k} \sum_{i=1}^{n} \epsilon_{\left(k / R_{i}^{1,2}, k / R_{i}^{2,2}\right)}(\cdot) \Rightarrow \tilde{\nu}^{\sqcap}(\cdot) \quad(k \rightarrow \infty, n / k \rightarrow \infty) .
$$

The verification of (3.16) follows the steps used in the proof of Proposition 2.9. Changing coordinate system in (3.16) leads to an estimator of $\nu_{1} \times S^{\sqcap}(\cdot)$ from the convergence in $M_{+}((0, \infty] \times[0, \infty])$

$$
\widehat{\nu_{1} \times S}{ }_{n}(\cdot):=\frac{1}{k} \sum_{i=1}^{n} \epsilon_{\left(k / R_{i}^{2,2}, R_{i}^{2,2} / R_{i}^{1,2}\right)}(\cdot) \Rightarrow \nu_{1} \times S^{\sqcap}(\cdot) \quad(k \rightarrow \infty, n / k \rightarrow \infty)
$$

and this produces an estimator of $S^{\sqcap}$ since in $M_{+}([0, \infty])$,

$$
\widehat{S_{n}^{\Pi}}(\cdot):=\frac{\sum_{i=1}^{n} \epsilon_{\left(k / R_{i}^{2,2}, R_{i}^{2,2} / R_{i}^{1,2}\right)}([1, \infty] \times \cdot)}{\sum_{i=1}^{n} \epsilon_{k / R_{i}^{2,2}}([1, \infty])} \Rightarrow S^{\sqcap}(\cdot) \quad(k \rightarrow \infty, n / k \rightarrow \infty) .
$$

This estimator may be modified as in (2.31) using the continuous bijection $T R:[0, \infty] \mapsto[0,1]$ defined by $T R: x \mapsto x /(1+x)$ to get in $M_{+}([0,1])$,

$$
\widehat{S_{n}^{\sqcap}}(\cdot) \circ T R^{-1} \Rightarrow S \sqcap \circ T R^{-1}(\cdot) .
$$

This summarizes how to obtain consistent estimators for extreme value index $\gamma^{\square}$ and the standardized hidden spectral measure $S \sqcap$.

\section{Detection of HDA}

Since HDA is a generalization of HRV, it is not surprising that the detection techniques have similarities to those used for HRV; see Mitra and Resnick [12] and Resnick [15, pages 316-340]. However, we deviate from the standard MEVT by assuming (2.1) instead of (1.2) and so proceed carefully. A first step is to detect the presence of asymptotic independence, which traditionally has been done with a density plot of a spectral measure after non-parametric transformation to Pareto 
scale [15, pages 316-321]. When asymptotic independence is present, we seek HDA. We consider how to define an appropriate spectral measure for detection of HDA.

Define $U^{(1)}(\cdot)=1 /\left(1-H^{(1)}(\cdot)\right.$, where $H^{(1)}$ is the distribution function of $X^{(1)}$. From (2.1) we get on $(0, \infty]^{2}$ that

$$
n P\left[\left(U^{(1)}\left(X^{1}\right) / n, U^{(1)}\left(X^{2}\right) / n\right) \in \cdot\right] \stackrel{v}{\rightarrow} \tilde{\nu}(\cdot),
$$

where $\tilde{\nu}(\cdot)$ is a Radon measure on $(0, \infty]^{2}$ related to the limit measure $\nu(\cdot)$ in (2.1) by

$$
\tilde{\nu}\left(\left(x^{1}, \infty\right] \times\left(x^{2}, \infty\right]\right)=\nu\left(\left(\psi^{\leftarrow}\left(x^{1}\right), \infty\right] \times\left(\psi^{\leftarrow}\left(x^{2}\right), \infty\right]\right) \quad\left(\boldsymbol{x} \in(0, \infty]^{2}\right),
$$

and $\psi$ is defined in (2.4) as $\psi(y):=\nu\left(\left\{\mathbf{z} \in \mathbb{E}: z^{(1)}>y\right\}\right)$. The measure $\tilde{\nu}(\cdot)$ satisfies the scaling

$$
\tilde{\nu}(c \cdot)=c^{-1} \tilde{\nu}(\cdot), \quad c>0,
$$

and convergence in (4.1) is equivalent to

$$
n P\left[\left(\frac{U^{(1)}\left(X^{(1)}\right)}{n},\left(\frac{U^{(1)}\left(X^{1}\right)}{U^{(1)}\left(X^{(1)}\right)}, \frac{U^{(1)}\left(X^{2}\right)}{U^{(1)}\left(X^{(1)}\right)}\right)\right) \in \cdot\right] \stackrel{v}{\rightarrow} \nu_{1} \times S(\cdot),
$$

on $(0, \infty] \times \delta \aleph^{(1)}$, where $\nu_{1}((y, \infty])=y^{-1}$ for $y>0, \delta \aleph^{(1)}=\left\{\boldsymbol{x} \in(0, \infty]^{2}: x^{(1)}=1\right\}$ and $S$ is a probability measure on $\delta \aleph^{(1)}$. The standardized spectral measure $S$ is related to $\tilde{\nu}$ in (4.1) by

$$
\tilde{\nu}\left(\left\{\boldsymbol{x} \in(0, \infty]^{2}: x^{(1)} \geq r, \boldsymbol{x} / x^{(1)} \in \Lambda\right\}\right)=r^{-1} S(\Lambda), \quad r>0 \text {, Borel set } \Lambda \subset \delta \aleph^{(1)} .
$$

To estimate this measure $S(\cdot)$, we define variants of the anti-ranks

$$
R_{i}^{1,(1)}=\left|\left\{j: X_{j}^{(1)} \geq X_{i}^{1}\right\}\right| \quad \text { and } \quad R_{i}^{2,(1)}=\left|\left\{j: X_{j}^{(1)} \geq X_{i}^{2}\right\}\right| \quad(1 \leq i \leq n) .
$$

A consistent estimator of $S(\cdot)$ is obtained from the convergence in $M_{+}\left(\delta \aleph^{(1)}\right)$

$$
\hat{S_{n}}:=\frac{\sum_{i=1}^{n} \epsilon\left(\frac{k}{R_{i}^{1,(1)} \wedge R_{i}^{2,(1)}},\left(\frac{R_{i}^{1,(1)} \wedge R_{i}^{2,(1)}}{R_{i}^{1,(1)}}, \frac{R_{i}^{1,(1)} \wedge R_{i}^{2,(1)}}{R_{i}^{2,(1)}}\right)\right)^{([1, \infty] \times \cdot)}}{\sum_{i=1}^{n} \epsilon \frac{k}{R_{i}^{1,(1)} \wedge R_{i}^{2,(1)}}([1, \infty])} \Rightarrow S(\cdot),
$$

$(k \rightarrow \infty, n / k \rightarrow \infty)$. The continuous bijection $T: \delta \aleph^{(1)} \mapsto[0,1]$ given by $T: \boldsymbol{x} \mapsto x^{2} /\left(x^{1}+x^{2}\right)$ transforms (4.7) to $\hat{S_{n}} \circ T^{-1} \Rightarrow S \circ T^{-1}(\cdot)$ in $M_{+}([0,1])$. A density plot of $\hat{S_{n}} \circ T^{-1}$ is easier to analyze because $[0,1]$ is a nicer space than $\delta \aleph^{(1)}$.

Analyzing the density plot using the points of $\hat{S}_{n} \circ T^{-1}$ should yield evidence falling into the following categories:

(i) The distribution $S \circ T^{-1}$ concentrates near 0 , so remove $\left\{(x, y) \in \mathbb{R}^{2}: y=-\infty\right\}$ and seek HDA on $\mathbb{E}^{\square}=[-\infty, \infty] \times(-\infty, \infty]$ or its first quadrant analogue, depending on the distribution of the second component of the random vector; see Remark 3.2 (2).

(ii) The distribution $S \circ T^{-1}$ concentrates near 1 , so remove $\left\{(x, y) \in \mathbb{R}^{2}: x=-\infty\right\}$ and seek HDA on $(-\infty, \infty] \times[-\infty, \infty]$ or its first quadrant analogue depending on the distribution of the first component of the random vector.

(iii) The distribution $S \circ T^{-1}$ concentrates near 0 and 1 , so remove $\left\{(x, y) \in \mathbb{R}^{2}: x=-\infty\right\} \cup$ $\left\{(x, y) \in \mathbb{R}^{2}: y=-\infty\right\}$ and seek HDA on $\mathbb{E}^{0}=(-\infty, \infty] \times(-\infty, \infty]$ or its first quadrant analogue, depending on the distribution of the smallest component of the random vector; see Remark 2.2 (2).

(iv) The distribution $S \circ T^{-1}$ does not have any of the above properties; we have no evidence for asymptotic independence and we do not consider HDA. 
We summarize our detection strategy and for concreteness assume $S \circ T^{-1}$ satisfies category (i): Check whether $X^{2}$ belongs to some maximal domain of attraction using a Hill or Pickands plots. If so, conclude HDA exists on $\mathbb{E}^{\sqcap}$. Then consider whether HDA exists also on $\mathbb{E}^{0}$ by examining a similar kernel density plot formed by using the points of the estimator of $S^{\sqcap} \circ(T R)^{-1}$ given in (3.19).

\section{Conclusion}

We defined HDA as a generalization of HRV and have shown by example that for some random vectors, HDA exists but HRV does not. Using similar methods as in HRV, we outlined detection and estimation methods for HDA. These methods are given to show what is possible and to emphasize there is a gap that such methods can fill to provide improved estimates of probability of simultaneous exceedance by components of a risk vector. However, we have not implemented the methods nor demonstrated utility by analyzing data. This will come in the future.

Our discussion here is restricted to two-dimensions. As observed for HRV [12], extensions to higher dimensions are not always straightforward and involve subtleties. In particular, in higher dimensions there are many more ways domains of attraction could be hidden and many more subspaces to explore for behavior that helps to estimate risk probabilities.

As with HRV [12], our detection and estimation methods are exploratory and our estimators are only provably consistent. More formal statistical theory is needed to turn exploratory methods into confirmatory ones.

\section{REFERENCES}

[1] A. A. Balkema and L. de Haan. On R. von Mises' condition for the domain of attraction of $\exp \left(-e^{-x}\right)^{1}$. Ann. Math. Statist., 43:1352-1354, 1972. ISSN 0003-4851.

[2] P. Billingsley. Convergence of Probability Measures. John Wiley \& Sons Inc., New York, second edition, 1999. ISBN 0-471-19745-9. A Wiley-Interscience Publication.

[3] J.T. Bruun and J.A. Tawn. Comparison of approaches for estimating the probability of coastal flooding. J. R. Stat. Soc., Ser. C, Appl. Stat., 47(3):405-423, 1998.

[4] L. de Haan. A characterization of multidimensional extreme-value distributions. Sankhyā Ser. A, 40(1):85-88, 1978. ISSN 0581-572X.

[5] L. de Haan and J. de Ronde. Sea and wind: multivariate extremes at work. Extremes, 1(1): 7-46, 1998.

[6] L. de Haan and A. Ferreira. Extreme Value Theory: An Introduction. Springer-Verlag, New York, 2006.

[7] J.E. Heffernan and S.I. Resnick. Hidden regular variation and the rank transform. Adv. Appl. Prob., 37(2):393-414, 2005.

[8] J.E. Heffernan and S.I. Resnick. Limit laws for random vectors with an extreme component. Ann. Appl. Probab., 17(2):537-571, 2007. ISSN 1050-5164. doi: 10.1214/105051606000000835.

[9] Xin Huang. Statistics of Bivariate Extreme Values. Ph.D. thesis, Tinbergen Institute Research Series 22, Erasmus University Rotterdam, Postbus 1735, 3000DR, Rotterdam, The Netherlands, 1992.

[10] A.W. Ledford and J.A. Tawn. Statistics for near independence in multivariate extreme values. Biometrika, 83(1):169-187, 1996. ISSN 0006-3444.

[11] A.W. Ledford and J.A. Tawn. Concomitant tail behaviour for extremes. Adv. in Appl. Probab., 30(1):197-215, 1998. ISSN 0001-8678.

[12] A. Mitra and S.I. Resnick. Hidden Regular Variation: Detection and Estimation. Arxiv preprint arXiv:1001.5058, 2010. 
[13] S.-H Poon, M. Rockinger, and J. Tawn. Modelling extreme-value dependence in international stock markets. Statist. Sinica, 13(4):929-953, 2003. ISSN 1017-0405. Statistical applications in financial econometrics.

[14] S.I. Resnick. Hidden regular variation, second order regular variation and asymptotic independence. Extremes, 5(4):303-336 (2003), 2002. ISSN 1386-1999.

[15] S.I. Resnick. Heavy Tail Phenomena: Probabilistic and Statistical Modeling. Springer Series in Operations Research and Financial Engineering. Springer-Verlag, New York, 2007. ISBN: $0-387-24272-4$.

[16] S.I. Resnick. Extreme Values, Regular Variation and Point Processes. Springer, New York, 2008. ISBN 978-0-387-75952-4. Reprint of the 1987 original.

[17] R.L. Smith. Statistics of extremes, with applications in environment, insurance and finance. In B. Finkenstadt and H. Rootzén, editors, SemStat: Seminaire Europeen de Statistique, Exteme Values in Finance, Telecommunications, and the Environment, pages 1-78. Chapman-Hall, London, 2003.

Abhimanyu Mitra, School of OR\&IE, Cornell University, Ithaca, NY-14853

E-mail address: am492@cornell.edu

Sidney I. Resnick, School of OR\&IE, Cornell University, Ithaca, NY-14853

E-mail address: sir1@cornell.edu 